\title{
Symposium
}

\section{Die strukturellen Ursachen sozialer Ungleichheit}

Symposiumsbeitrag zu: Michael Hartmann, Die Abgehobenen. Wie die Eliten die Demokratie gefährden. Frankfurt a.M./New York: Campus 2018, 276 S., gb., $19,95 €$

Besprochen von Prof. Dr. Jens Beckert: Direktor am Max-Planck-Institut für Gesellschaftsforschung Köln, E-Mail: Beckert@mpifg.de

https://doi.org/10.1515/srsr-2020-0002

Schlüsselwörter: Eliten, soziale Ungleichheit, Reichtum

Michael Hartmann ist der prominenteste Elitenforscher in Deutschland. Seit den 1990er Jahren erforscht der Darmstädter Soziologe Eliten und insbesondere die Mechanismen ihrer Reproduktion. Wie kommt es, dass die Sprösslinge der sozialen Oberschicht mit viel höherer Wahrscheinlichkeit selbst $\mathrm{zu}$ dieser sozialen Klasse gehören werden als die Kinder unterer oder mittlerer sozialer Schichten? Gerade für Deutschland ist die Antwort auf diese Frage nicht trivial, fehlen doch die klar auf die Elite zugeschnittenen Bildungsinstitutionen, die etwa für die USA, für England und für Frankreich charakteristisch sind. Und auch Privatschulen spielen, wie Hartmann in dem zu besprechenden Buch schildert (8), in Deutschland eine vergleichsweise geringe Rolle, der medialen Aufmerksamkeit für das Internat Schloss Salem und anderer Reichenschulen zum Trotz.

Die Abgehobenen ist kein Buch, das neue Forschungsergebnisse präsentiert. Es ist vielmehr eine flott geschriebene Zusammenfassung der langjährigen Forschungen von Hartmann, die hier für ein breites Publikum aufbereitet wurden. Das Buch ist gut geschrieben, liest sich sehr flüssig und geradezu spannend. Hartmann hält den Leser über 250 Seiten in einer Empfindung der Empörung, sind die präsentierten Sachverhalte doch in den Augen des Autors skandalös. Entgegen unserem gängigen und politisch auch immer gepriesenen Selbstverständnis einer Leistungsgesellschaft, in der Chancen gerecht verteilt sind und die jedem, der sich nur genügend anstrengt und das Talent mitbringt, den Weg nach oben offenhält, verweist Hartmann darauf, dass dies allenfalls eine politisch bequeme Ideologie ist. Wer nicht in die Oberschicht hineingeboren wurde, kämpft auf einem 
unebenen Spielfeld, wer in die soziale Oberschicht geboren wurde, dem wird es leicht gemacht, bekommt er doch ganz zwanglos von Eltern und Bildungsinstitutionen den richtigen Habitus und die notwendigen Kontakte vermittelt, die ihn nach oben befördern werden. Noch dazu verfolgen die Eliten ihre Interessen rücksichtslos, auch am Rande der Legalität und manchmal mit illegalen Mitteln. Ausführlich erfährt der Leser, welche Reichen und deren Berater bereits in Untersuchungshaft saßen oder Haftstrafen verbüßten.

Die Abgehobenen beschreibt die sozialstrukturelle Herkunft von Eliten in Deutschland und in einer vergleichenden Perspektive insbesondere die Situation in England, Frankreich und den USA. Das Buch zeichnet außerdem die Mechanismen der Selektion nach, die zur Selbstreproduktion der Eliten führt. Darüber hinaus wird, anhand von Anekdoten und den Ergebnissen von Umfragen, die Dis$\operatorname{tanz}$ aufgezeigt, die im Hinblick auf Einstellungen und Problemwahrnehmungen zwischen denen „da oben“ und dem Rest der Bevölkerung besteht. Nichts von dem, was berichtet wird, überrascht den Leser eigentlich, es ist, was man ohnehin schon immer, informiert über die Berichterstattung von Skandalen, vermutet hat und hier anhand umfangreicher deskriptiver Statistiken und vielfältiger Geschichten en Detail aufbereitet bekommt.

All dies ist soziologische Aufklärung im besten Sinn. Hartmann betreibt eine Soziologie, die sich nicht durch die Verwendung avancierter Methoden zu beweisen versucht, sondern durch das emsige Zusammentragen von Informationen und Geschichten, um grundlegende Mechanismen der Reproduktion sozialer Ungleichheit gut verständlich darzulegen. Wir sind anders, als die gängige Ideologie es behauptet. Und mit der Beschreibung von Hartmann bekommen wir die Bestätigung eines immer schon vorhanden gewesenen Gefühls, dass nicht Leistungen, sondern Privilegien über die soziale Stratifikation entscheiden.

Dieses Vorgehen von Hartmann birgt allerdings auch seine Gefahren. Das Bild, das Die Abgehobenen von unserer Gesellschaft zeichnet, ist möglicherweise zu einfach und die Phänomene widersprüchlicher, als Hartmann dies zugesteht. Um dies zu erkennen, genügt die genaue Lektüre des Buches.

Hartmanns These besteht darin, dass die Elite nicht nur abgehoben ist, sondern auch immer weiter abhebt und dass hierin die Ursache für sich perpetuierende soziale Ungleichheit und - aktuell - die mit dem Begriff des Populismus umschriebenen politischen Fragmentierungsprozesse zu suchen ist. „Eine zunehmend geschlossene Gesellschaft“ ist das zweite Kapitel überschrieben. Die Befunde des Buches sind jedoch zumindest ein wenig bunter. Als ironisch zu werten ist vermutlich, dass der Buchumschlag des Campus Verlags einen Golfspieler unter einer Glasglocke zeigt, der Leser des Buches jedoch erfährt, dass die Elite gar nicht Golf spielt (zu zeitaufwändig), sondern wandern oder joggen geht (72). Auch zeigen die Daten von Hartmann, dass sich die Elite durchaus nicht zunehmend abschließt, sondern es, 
insbesondere in England, aber auch in den USA, durchaus Prozesse der Öffnung gibt. In Deutschland ist die Elite ohnehin weniger homogen als in den Vergleichsländern (96). Kam noch vor wenigen Jahrzehnten ein Großteil der Britischen Elite aus einer Handvoll Public Schools und den Universitäten in Oxford und Cambridge, so hat sich dies heute pluralisiert. Lediglich im zentralistischen, auf den Großraum Paris und ein paar Eliteinstitutionen konzentrierten Frankreich lässt sich von einer geschlossenen Elite sprechen.

Brüchig erscheint auch die das Buch strukturierende These, dass soziale $\mathrm{Zu}$ gehörigkeit umstandslos die politische Positionierung bestimmt. Nur so lässt sich verstehen, weshalb Hartmann ein Problem darin erkennt, dass Minister zunehmend aus der Oberschicht und nicht mehr wie früher, zu einem guten Teil aus der Arbeiterschicht kommen. Die „eigene Lage und Herkunft [vernebelt] den Blick auf die gesellschaftliche Realität breiter Bevölkerungskreise“ (14). Das Sein bestimmt das Bewusstsein. Für eine Korrespondenz zwischen sozialer Position und politischem Handeln gibt es zwar allerhand Belege, doch auch auffällige Abweichungen, die von Hartmann beschrieben werden, nicht jedoch zur Veränderung der Ausgangsthese führen. Die Sozialdemokratie des Dritten Weges in den neunziger Jahren, auf deren Konto die wesentlichen Reformen in Richtung Neoliberalismus gehen, wurden sämtlich durch soziale Aufsteiger bestimmt: Schröder und Clinton kommen aus einfachsten sozialen Verhältnissen, Blair entstammt einem höheren sozialen Milieu. Ähnliches gilt auch für die erste Generation konservativer Reformer in den 1980er Jahren, Maggie Thatcher und Ronald Reagan (139). Warum haben diese Politiker nicht ihren „Klassenstandpunkt“ vertreten, als sie einmal an der Macht waren? Andererseits wurden die zentralen Sozialreformen des 20. Jahrhunderts in den USA von Personen aus den höchsten Elitekreisen durchgesetzt: den beiden Roosevelts und den Kennedys. Dies soll nicht grundlegend in Abrede stellen, dass die Zusammensetzung der politischen Elite für die getroffenen politischen Entscheidungen bedeutungslos wäre, doch scheint der Zusammenhang vielschichtiger als von Hartmann suggeriert. Lässt sich politische Positionierung wirklich auf soziale Herkunft reduzieren?

Problematisch erscheint auch, dass die Elitenreproduktion bei Hartmann als wenig subtil erscheint. Die Elite hält die anderen von sich fern, indem sie ein feines Gespür für habituelle Unterschiede hat und dies bei Personalentscheidungen zur Ausgrenzung nutzt. Die nuancierten Mechanismen entgehen Hartmann. In den USA hat Shamus Khan (2011 und Accominotti et al. 2018) etwa anhand der Untersuchung einer privaten Eliteschule und der New Yorker Philharmoniker gezeigt, dass die Mechanismen der Elitenreproduktion sehr viel spitzfindiger sein können. Die gesellschaftliche Missachtung des Ausschlusses von Minderheitengruppen bringt Eliteinstitutionen in eine Legitimationskrise, auf die reagiert wird, indem neuen Gruppen Zugang gewährt wird. Doch zugleich werden interne Me- 
chanismen der Exklusion geschaffen, durch die sich der innere Zirkel auch weiterhin abgrenzen kann.

Dies führt zu der vielleicht wichtigsten Einschränkung des Buches von Hartmann. Soziale Ungleichheit wird von ihm letztendlich mit der sozialen Herkunft der Machthabenden erklärt. Herkunft führe zu einem Habitus und zu sozialen Netzwerken, die zusammen geeignet sind, die Emporkömmlinge auf Distanz zu halten und Politik für die Eliten zu betreiben. Das Problem sozialer Ungleichheit sind Die Abgehobenen. Dabei bleiben die institutionellen und strukturellen Faktoren außen vor, die Erklärungen sozialer Ungleichheit in der politischen Ökonomie bestimmen. Dass die Liberalisierung von Märkten (Globalisierung) das Kräfteverhältnis zwischen mobilem Kapital und immobiler Arbeit zugunsten der Kapitalbesitzer verschoben hat; dass die Tertialisierung der Wirtschaft die Organisationsmacht der Gewerkschaften reduziert hat; dass die Mobilität von Kapital bei gleichzeitig fehlender transnationaler Regulationsmacht zur Reduzierung von Kapitalertragssteuern und anderer Vermögenssteuern führt; dass die Flexibilisierung von Arbeitsmärkten zu diskontinuierlichen Erwerbsbiografien in der Mittelschicht führt und diese in ihrem sozialökonomischen Status bedroht; dass die Privatisierung höherer Bildung sozial ungleiche Bildungschancen hervorruft; dass die Reduzierung sozialstaatlicher Leistungen und stagnierende Löhne in vielen Ländern - etwa in den USA und in England - eine massive Erhöhung privater Verschuldung zeitigt; dass Kapitalbesitzer eine höhere Rendite auf ihr Kapital erhalten als die Kleinanleger. Etc., etc. Natürlich kennt Hartmann all diese Befunde und teilweise werden sie von ihm auch erwähnt, doch die strukturellen Veränderungen des globalisierten Kapitalismus finden keinen systematischen Eingang in seine Erklärung sozialer Ungleichheit, die sich allein auf die soziale Herkunft der Eliten stützt.

Dies ist nicht nur für die Analyse folgenreich, sondern auch für die politischen Schlussfolgerungen im letzten Kapitel des Buches. Hartmann gibt sich etwa für die Britische Labour Party unter Jeremy Corbyn hoffnungsfroh, weil in dessen Schattenkabinett die Arbeiterschicht wieder viel stärker vertreten ist. Auch sympathisiert er mit den südeuropäischen populistischen Bewegungen, weil diese zur Rekrutierung einer neuen (weniger abgehobenen) politischen Elite aus sozialen Bewegungen führten. Für die Möglichkeit eines Politikwechsels führt er zaghafte politische Schritte wie die Regulierung von Airbnb in vielen Großstädten oder den automatischen Datenaustausch über die Finanzkonten von Ausländern an (245). Da die strukturellen Bedingungen des heutigen Kapitalismus bei ihm keinen systematischen Eingang finden, bleiben die Vorschläge persönliche Präferenzen und Hoffnungen, denen eine Analyse von strukturellen Kräftefaktoren fehlt. Möglicherweise wird die Demokratie nicht so sehr von ihren Eliten gefährdet als vom Kapitalismus in seinem heutigen Zuschnitt. 
Die Stärke von Hartmanns Buch besteht in der Beschreibung der sozialen $\mathrm{Zu}$ sammensetzung unterschiedlicher Elitegruppen in der Gesellschaft und einiger der Mechanismen ihrer Reproduktion. Die Befunde sind aus Hartmanns früheren Büchern bereits bekannt und in der Soziologie vielfältig erforscht. Für die Erklärung der Entwicklung sozialer Ungleichheit in der Gesellschaft benennt Die Abgehobenen allenfalls einen Teilaspekt.

\section{Literatur}

Accominotti, F.; Khan, S.; Storer, A. How Cultural Capital Emerged in Gilded Age America: Musical Purification and Cross-Class Inclusion at the New York Philharmonic. American Journal of Sociology 2018, 123, 1743-1783.

Khan, S. Privilege: The Making of an Adolescent Elite at St. Paul's School; Princeton University Press: Princeton, 2011. 